\title{
Comparación antropométrica de un grupo de ciclistas de ruta y pista*
}

\author{
Henry Humberto León Ariza** \\ Daniel Alfonso Botero Rosas ${ }^{* * *}$ \\ Carlos Enrique Melo**** \\ Javier Porras Álvarez ${ }^{* * * * *}$
}

Recibido: diciembre 11 de 2013 • Evaluado: enero 30 de 2014

Aceptado: febrero 14 de 2014

\section{Resumen}

La antropometría y la composición corporal constituyen una parte fundamental en la evaluación de los deportistas; en Colombia no hay reporte de evaluaciones comparativas de la composición corporal entre ciclistas de pista y de ruta. Quince ciclistas profesionales de género masculino ( 9 de ruta y 6 de pista) fueron evaluados antropométricamente; se determinaron: peso, talla, índice de masa corporal (IMC), somatotipo y composición

"Artículo de investigación científica de una investigación desarrollada entre marzo de 2012 y marzo de 2013, con el apoyo del Laboratorio de Fisiología de la Universidad de La Sabana y Jenaro Sport Lab.

"* Licenciado en Educación Física, médico cirujano y doctorando en Biociencias. Docente investigador de la Facultad de Medicina de la Universidad de La Sabana. Correo: henrylear@ clinicaunisabana.edu.co

*** Médico cirujano, magíster en Ingeniería Biomédica, doctor en Ingeniería Biomédica. Docente investigador de la Facultad de Medicina de la Universidad de La Sabana y la Universidad Antonio Nariño. Correo: danybotero@hotmail.com

**** Médico cirujano, magíster en Fisiología Humana. Correo: kiek.dr@gmail.com

***** Licenciado en Educación Física, magíster en Ciencias de la Actividad Física y el Deporte, doctorando en Medicina Deportiva. Docente investigador de la Universidad Pedagógica y Tecnológica de Colombia. Correo: japadbco@gmail.com 
corporal porcentual de grasa, músculo y hueso, con el objetivo de establecer diferencias estadísticamente significativas entre los grupos. Se obtuvo como resultado un mayor peso e IMC en los ciclistas de pista; además, los pliegues y perímetros fueron superiores comparados con los de ciclismo de ruta, exhibiendo un mayor porcentaje promedio de grasa $(8,6 \% \pm 0,7$ pista y $7,5 \%$ $\pm 0,4$ ruta $\mathrm{p}<0,05)$ y músculo $(54,8 \% \pm 3,4$ pista y $51,5 \% \pm 2,3$ ruta $\mathrm{p}<$ $0,05)$. El somatotipo de los ciclistas de pista $(2,7-5,3$ - 1,9), en comparación con el de los ciclistas de ruta $(2,0$ - 4,1 - 2,8), muestra un mayor predominio endomórfico y mesomórfico y un valor ectomórfico inferior, con una distancia entre la dispersión de los somatotipos estadísticamente significativa $(3,67)$. La diferencia en las demandas energéticas para cada grupo de ciclistas se hace evidente en las adaptaciones a nivel de la composición corporal, resultados similares a los publicados en otros estudios.

Palabras clave: Ciclismo de pista, ciclismo de ruta, composición corporal, somatotipo. 


\section{Anthropometric comparison of a group of track and route cyclists}

\section{Abstract}

Anthropometric and corporal composition is an essential part in the evaluation of athletes, in Colombia there is no knowledge of benchmarking body composition between track and road cyclists. 15 male professional cyclists (9 route and 6 track), were evaluated, were determined: weight, height, body mass index (BMI), body composition percentage of fat, muscle and bone and somatotype, with the goal of finding statistically significant differences between groups. The result showed a higher weight and BMI in track cyclists; in addition, folds and circumferences were higher than those found in road cyclists constituting a higher percentage of fat $(8.6 \% \pm 0.7$ track and $7.5 \% \pm 0.4$ route $\mathrm{p}<0.05)$ and muscle $(54.8 \pm 3.4 \%$ track and $51.5 \% \pm 2.3$ route $\mathrm{p}<0.05)$. The somatotype of track cyclists $(2.7-5.3-1.9)$ compared to road cyclists $(2.0-4.1-2.8)$, shows a predominance endomorphic and mesomorphic and less ectomorphism value with a distance between the dispersion of the somatotypes statistically significant (3.67). The difference in energy demands for each group of riders is evident in the changes in body composition, found similar results to those reported in other studies.

Keywords: Track cycling, route cycling, corporal composition, somatotype. 


\section{Introducción}

El proceso de evaluación del rendimiento y la caracterización deportiva constituye, en la actualidad, el resultado del análisis de múltiples variables, entre estas el comportamiento fisiológico del atleta, el análisis biomecánico, y la composición y distribución corporal, entre otras (Reilly, Morris \& Whyte, 2009).

Las evaluaciones antropométricas y de la composición corporal juegan un papel importante en el proceso de análisis del rendimiento deportivo (Bayio et al., 2006; Foley, Bird \& White, 1989; Hoffman, 2008; Martínez et al., 2010; Pradas et al., 2007), debido a la estrecha relación observada entre las características antropométricas y los resultados de las diferentes modalidades deportivas (Caruso et al., 2009; Carter, 2003). Además, el estudio antropométrico y de la composición corporal constituyen un método adecuado en la evaluación nutricional de los atletas (Holway \& Spriet, 2011)..

En las modalidades de ruta y pista del ciclismo, la composición corporal juega un papel importante; sin embargo, las investigaciones al respecto son limitadas. Carter (1982), con motivo de los Juegos Olímpicos de Montreal 1976, publicó los resultados del somatotipo para ciclistas, sin distinción de modalidad deportiva. En 1982, se publicaron los primeros trabajos de composición corporal en ciclistas, diferenciando las modalidades de ruta y pista en el equipo olímpico inglés, los cuales mostraron diferencias en la composición corporal (White et al., 1982a; White et al., 1982b).

En Colombia, no se han encontrado publicaciones que comparen los resultados de composición corporal de ciclistas de pista versus ciclistas de ruta, por lo que el presente trabajo se convierte en la primera investigación de este tipo.

La evolución en el entrenamiento, la comprensión de la fisiología aplicada del ejercicio, su relación con los límites fisiológicos y el alto nivel de investigación en rendimiento deportivo, obligan al análisis de los datos antropométricos como elemento fundamental en la construcción de la maestría deportiva. 


\section{Método}

Participantes. En el estudio, participaron 15 ciclistas de sexo masculino (9 ciclistas de ruta y 6 ciclistas de pista) pertenecientes a la Liga de Ciclismo de Boyacá (Colombia).

Instrumentos. Todas las mediciones antropométricas fueron realizadas en horas de la mañana por el mismo evaluador certificado por la International Society for the Advancement of Kinanthropometry (ISAK) nivel I, y siguiendo las recomendaciones de la Sociedad Internacional de Antropometría (Lohman, Roche \& Martorell, 1991). El peso corporal se midió utilizando una báscula Full Body Sensor Body Composition Monitor, con una capacidad de 330 libras y graduación de 0,2 libras; la talla se determinó con un estadiómetro Health o meter ${ }^{\circledR}$, con un rango de medición de lectura directa en el contador de 1-220 cm; los pliegues cutáneos (bíceps, tríceps, subescapular, suprailiaco, muslo y pantorrilla) se midieron con un calibrador Harpenden Body Scale/Body Care, con un rango de medición de 0-80 mm y una precisión del $99.0 \%$; los perímetros (brazo, muslo, pantorrilla, abdominal y tórax) se midieron con cinta antropométrica metálica, con capacidad de medición de 0-150 cm, y los diámetros (biestiloideo, biepicondileo, bicondileo y bimaleolar) se midieron con un antropómetro calibrador Lafayette, con un rango de medición de 0-60 $\mathrm{mm}$ de precisión.

Procedimiento. El cálculo del IMC se determinó usando la fórmula propuesta por Quetelet en 1832 (Eknoyan, 2008); el porcentaje de grasa se calculó mediante la fórmula de Yuhasz para deportistas (Yuhasz, 1974); el porcentaje de masa muscular se calculó con la fórmula de Doupe (Doupe et al., 2010); el porcentaje de peso óseo se calculó con la fórmula de Von Döbeln, modificada por Rocha (Rocha, 1975); y el somatotipo se determinó mediante el método Heath-Carter (Carter, 2002).

Análisis estadístico. Los datos se presentaron en forma de promedios con sus desviaciones estándar; la normalidad se verificó con la prueba de Kolmogorov-Smirnov; el análisis de las diferencias entre los grupos se realizó mediante la prueba de T-student para datos no pareados con diferencia de varianzas y se estableció como valor de significancia $\mathrm{p}<0,05$. El análisis del somatotipo se llevó a cabo siguiendo el modelo propuesto por Carter (1982), en el cual se calcula: la distancia de dispersión somatotípica (SDD) y el índice de dispersión somatotípica (SDI); para los valores del SDD o del 
SDI, se considera estadísticamente significativo un valor igual o superior a 2 unidades (Esparza \& Alvero, 1993).

\section{Resultados}

La tabla 1 presenta los datos generales de los ciclistas evaluados. Se encontraron diferencias estadísticamente significativas en el peso y el IMC, los cuales son superiores en los ciclistas de ruta.

Los valores de los pliegues cutáneos y los perímetros tomados fueron superiores en los ciclistas de pista en relación con los ciclistas de ruta, con diferencias estadísticamente significativas entre la gran mayoría de los datos; por ejemplo, el perímetro del muslo tanto medio como máximo alcanza una diferencia cercana a los $5 \mathrm{~cm}$. Los diámetros óseos son bastante similares. Los valores de pliegues, perímetros y diámetros se encuentran en la tabla 2; la comparación de los pliegues, en la figura 1 y la comparación de perímetros, en la figura 2.

Hubo diferencias en la composición corporal entre los dos grupos. El porcentaje de grasa fue mayor en los ciclistas de pista que en los ciclistas de ruta $(8,6 \% \pm 0,7$ y $7,5 \% \pm 0,4, p<0,05)$; de la misma forma, el porcentaje de masa muscular también fue superior en los ciclistas de pista $(54,8 \% \pm$ $3,4$ y $51,5 \% \pm 2,3, \mathrm{p}<0,05)$ y el componente residual fue mayor en los ciclistas de ruta $33,9 \% \pm 2,9$. No se presentó diferencia significativa en el componente óseo. Los resultados se presentan en la figura 3.

El somatotipo de los ciclistas también presentó diferencias estadísticamente significativas. El componente endomórfico fue mayor en los ciclistas de pista que en los ciclistas de ruta $(2,67 \pm 0,42$ y $2,01 \pm 0,28, \mathrm{p}<0,05)$, el componente mesomórfico también fue superior $(5,25 \pm 0,96$ y $4,06 \pm 0,70$, $\mathrm{p}<0,05)$, mientras que el componente ectomórfico fue más bajo en los ciclistas de pista $(1,91 \pm 0,66$ y $2,75 \pm 0,64, \mathrm{p}<0,05)$. El SDI, representativo de la dispersión de los datos en cada uno de los grupos, es superior para los ciclistas de pista (SDI $=2,6$ ) en comparación con el de los ciclistas de ruta $(\mathrm{SDI}=2,0)$. Igualmente, la distancia de dispersión del somatotipo (SDD), o la distancia que separa a ambos somatotipos, también es estadísticamente significativa ( $\mathrm{SDD}=3,67$ ). Los resultados del somatotipo se encuentran en las figuras 4 y 5 . 


\section{Discusión}

Los datos de talla y peso presentan un comportamiento similar al observado en otros estudios (Foley et al., 1989), en los cuales los deportistas de pista son más pesados, tienen un mayor IMC y son ligeramente más bajos de estatura, en comparación con los deportistas de ruta. De otra parte, la composición corporal demuestra diferencias interesantes, el menor porcentaje de grasa en deportistas de ruta está en relación con el predominio del metabolismo aeróbico de estos deportistas y una alta utilización de las grasas como fuente de energía (Lucia, Hoyos \& Chicharro, 2001; Sallet et al., 2006). Estos resultados asociados al menor porcentaje de grasa también han sido descritos previamente (Foley et al., 1989; McLean \& Parker, 1989).

Los perímetros de miembros superiores e inferiores para los ciclistas de pista fueron también superiores, al evidenciar que el comportamiento se presenta en los perímetros corregidos; es posible afirmar que dicho incremento corresponde al aumento de la masa muscular y no al tejido adiposo, corroborado por los valores superiores en el porcentaje de masa muscular; las pruebas de pista requieren una alta demanda del metabolismo anaeróbico, lo cual es característico de pruebas de corta duración (Craig \& Norton, 2001). De otra parte, las demandas de potencia muscular obligan a tener una buena masa muscular y un alto nivel de reclutamiento de fibras (Atkinson et al., 2003).

La no diferencia en los diámetros óseos se asocia a una estructura esquelética similar en ambas poblaciones, lo cual cuenta con un importante componente genético.

El somatotipo encontrado en los ciclistas analizados también es coherente con los resultados publicados previamente (Foley et al., 1989; White et al., 1982a; White et al., 1982b; McLean \& Parker, 1989; Martinez, Fideu, \& Ferrer, 1993, Padilla, Taylor, Yuhasz, \& Velázquez, 2004), en donde todos muestran un mayor predominio del componente mesomórfico y un menor componente ectomórfico para los ciclistas de pista, sin diferencias significativas entre los resultados encontrados y los publicados anteriormente, Tabla 3. Este patrón del somatotipo está en estrecha relación con un aumento de la masa funcional y se asocia a un aumento en el rendimiento durante actividades explosivas de los ciclistas de pista (Craig \& Norton, 2001; Atkinson et al., 2003), mientras que la menor ectomorfia en los ciclistas de ruta incrementa la masa funcional al reducir el tejido adiposo, lo 
cual le permite minimizar la resistencia del pedaleo y ofrecer una menor área frontal de superficie (Gregor, 2000).

\section{Conclusión}

La evaluación de la composición corporal y el desarrollo de la carta somatoscópica pueden ser considerados criterios valiosos dentro de los procesos de selección y direccionamiento de los deportistas, y constituyen, además, una herramienta práctica que contribuye a la planificación y al seguimiento deportivo de los ciclistas tanto de ruta como de pista.

\section{Referencias}

Atkinson, G., Davison, R., Jeukendrup, A., \& Passfield, L. (2003). Science and cycling: current knowledge and future directions for research. J Sports Sci, 21(9), 767-787. doi:10.1080/0264041031000102097

Bayios, I. A., Bergeles, N. K., Apostolidis, N. G., Noutsos, K. S., \& Koskolou, M. D. (2006). Anthropometric, body composition and somatotype differences of Greek elite female basketball, volleyball and handball players. J Sports Med Phys Fitness, 46(2), 271-280.

Carter, J. E. (1982). Somatotypes of Montreal olympic athletes. Physical structure of Olympic athletes Part 1. The Montreal Olympic Games anthropological project. Medicine and Sport 16, 53-80.

Carter, J. L. (2002). The Heath-Carter Anthropometric somatotype - Instruction manual. San Diego: Department of Exercise and Nutritional Sciences.

Carter, J. L. (2003). Factores morfológicos que limitan el rendimiento humano. PubliCE Standard Pid, 139.

Caruso, J. F., Ramey, E., Hastings, L. P., Monda, J. K., Coday, M. A., \& McLagan, J. (2009). Anthropometry as a predictor of high speed performance. Int J Sports Med, 30(7), 522-525.

Craig, N., \& Norton, K. (2001). Characteristics of Track Cycling. Sports Med, 31(7), 457-468.

Doupe, M. B., Martin, A. D., Searle, M. S., Kriellaars, D. J., \& Giesbrecht, G. G. (2010). A new formula for population-based estimation of whole body muscle mass in males. Can J Appl Physiol, 22(6), 598-608. 
Esparza, R. F., \& Alvero, J. R. (1993). Manual de cineantropometría. España: Federación Española de Medicina del Deporte (Fedeme).

Eknoyan, G. (2008). Adolphe Quetelet (1796-1874). The average man and indices of obesity. Nephrol Dial Trasplant, 23, 47-51.

Foley, J. P., Bird, S. R., \& White, J. A. (1989). Anthropometric comparison of cyclists from diferent events. Br J Sports Med, 23(1), 30-33.

Gregor, R. J. (2000). Biomechanics of cycling. Exercise and sport science. Filadelfia: Lippincott Williams and Williams.

Hoffman, M. D. (2008). Anthropometric characteristics of ultramarathoners. Int J Sports Med, 29(10), 808-811.

Holway, F. E., \& Spriet, L. L. (2011). Sport-specific nutrition: practical strategies for team sports. J Sports Sci, 29(Suppl 1), S115-S125. doi:10.1080/02640414 .2011 .605459

Lohman, T. G., Roche, A. F., \& Martorell, R. M. (1991). Anthropometric Standarization Reference Manual. Champaign: Human Kinetics Books.

Lucia, A., Hoyos, J., \& Chicharro, J. L. (2001). Physiology of professional road cycling. Sports Med., 31(5), 325-337.

Martínez, S., Pasquarelli, B. N., Romaguerra, D., Arasa, C., Tauler, P., \& Aguiló, A. (2010). Anthropometric characteristics and nutritional profile of young amateur swimmers. J Strength Cond Res, 25(4), 1126-1133.

Martínez, L., Fideu, M., \& Ferrer, V. (1993). Estudio cineantropométrico en 58 ciclistas de competición. Archivos de Medicina del Deporte, 10(38), 121-125.

McLean, B. D., \& Parker, A. W. (1989). An anthropometric analysis of elite Australian track cyclists. J Sports Sci, 7(3), 247-255. doi:10.1080/02640418908729845

Padilla, J., Taylor, A. W., Yuhasz, M. S. \& Velázquez, M. A. (2004). Algunas características antropométricas de una población de atletas mexicanos. Revista Médica del Hospital Geneneral de México, 67, 11-21.

Pradas, F., Carrasco, L., Martínez, E., \& Guerrero, P. (2007). Perfil antropométrico, somatotipo y composición corporal de jóvenes de tenis de mesa. Rev Int Cienc Deporte, 3, 11-23.

Reilly, T., Morris, T., \& Whyte, G. (2009). The specificity of training prescription and physiological assessment: a review. J Sports Sci, 27(6), 575-589. doi:910136194 [pii];10.1080/02640410902729741

Rocha, M. S. L. (1975). Peso ósseo do brasileiro de ambos os sexos de 17 a 25 años. Arq Anat Antrop, 1, 445-451. 
Sallet, P., Mathieu, R., Fenech, G., \& Baverel, G. (2006). Physiological differences of elite and professional road cyclists related to competition level and rider specialization. J Sports Med Phys Fitness, 46(3), 361-365.

White, J. A., Quinn, G., Al-Dawalibi, M., \& Mulhall, J. (1982a). Seasonal changes in cyclists' performance. Part I. The British Olympic road race squad. $\mathrm{Br} J$ Sports Med, 16(1), 4-12.

White, J. A., Quinn, G., Al-Dawalibi, M., \& Mulhall, J. (1982b). Seasonal changes in cyclists' performance. Part II. The British Olympic track squad. Br J Sports Med, 16(1), 13-21.

Yuhasz, M. S. (1974). Physiscal Fitness Manual. Ontario: Universidad de Western Ontario.

Tabla 1. Datos generales de los ciclistas evaluados

\begin{tabular}{llccccc}
\hline & & \multicolumn{2}{c}{ PISTA $\mathrm{n}=6$} & \multicolumn{2}{c}{ RUTA $\mathrm{n}=9$} & \\
\hline & & $\overline{\mathrm{X}}$ & $\mathrm{SD}$ & $\overline{\mathrm{X}}$ & $\mathrm{SD}$ & $p$ \\
\multirow{2}{*}{ Datos } & Talla $(\mathrm{cm})$ & 171,23 & 4,57 & 171,57 & 5,13 & 0,897 \\
generales & Edad & 22,83 & 3,87 & 19,00 & 1,66 & 0,060 \\
& Peso $(\mathrm{Kg})$ & 69,58 & 2,13 & 64,43 & 3,11 & $0,002^{*}$ \\
& IMC & 23,76 & 1,03 & 21,90 & 0,86 & $0,005^{*}$ \\
\hline
\end{tabular}

$\bar{X}=$ Promedio, SD: Desviación estándar, IMC: Índice de masa corporal; * = Diferencia estadísticamente significativa.

Fuente: autores, 2013 
Tabla 2: Datos antropométricos de los ciclistas evaluados

\begin{tabular}{|c|c|c|c|c|c|c|}
\hline & & \multicolumn{2}{|c|}{ PISTA $n=6$} & \multicolumn{2}{|c|}{ RUTA $n=9$} & \multirow[b]{2}{*}{$p$} \\
\hline & & $\bar{x}$ & SD & $\bar{x}$ & SD & \\
\hline \multirow{6}{*}{ Pliegues } & Tríceps (mm) & 7,33 & 1,03 & 6,12 & 0,90 & $0,042 *$ \\
\hline & Subescapular (mm) & 10,13 & 1,01 & 8,07 & 0,87 & $0,002 *$ \\
\hline & Abdominal (mm) & 10,77 & 2,13 & 7,60 & 2,97 & $0,032 *$ \\
\hline & Suprailiaco (mm) & 9,13 & 2,22 & 6,73 & 1,44 & $0,048 *$ \\
\hline & Muslo (mm) & 8,77 & 2,25 & 6,98 & 1,89 & 0,142 \\
\hline & Pierna $(\mathrm{mm})$ & 5,43 & 0,81 & 4,24 & 1,07 & $0,030 *$ \\
\hline \multirow{9}{*}{ Perímetros } & Brazo relajado $(\mathrm{cm})$ & 26,93 & 1,32 & 24,90 & 0,94 & $0,011 *$ \\
\hline & Brazo contraído $(\mathrm{cm})$ & 31,32 & 1,05 & 28,47 & 1,20 & $0,000 *$ \\
\hline & Abdominal $(\mathrm{cm})$ & 77,77 & 0,85 & 74,19 & 2,28 & $0,001 *$ \\
\hline & Muslo medio (cm) & 56,23 & 1,15 & 51,90 & 1,72 & $0,000 *$ \\
\hline & Muslo máximo (cm) & 57,30 & 1,43 & 52,63 & 1,41 & $0,000 *$ \\
\hline & Pierna contraído $(\mathrm{cm})$ & 36,28 & 1,80 & 33,76 & 1,62 & $0,020 *$ \\
\hline & Muslo corregido $(\mathrm{cm})$ & 54,43 & 1,57 & 50,52 & 1,77 & $0,001 *$ \\
\hline & Pierna corregido $(\mathrm{cm})$ & 34,58 & 1,72 & 32,42 & 1,64 & $0,035 *$ \\
\hline & Brazo corregido $(\mathrm{cm})$ & 24,63 & 1,13 & 22,98 & 0,87 & $0,014 *$ \\
\hline \multirow{4}{*}{ Diámetros } & Biepicodileo $(\mathrm{cm})$ & 7,02 & 0,31 & 6,74 & 0,22 & 0,096 \\
\hline & Biestiloideo $(\mathrm{cm})$ & 5,47 & 0,33 & 5,53 & 0,73 & 0,816 \\
\hline & Bicondilar $(\mathrm{cm})$ & 9,42 & 0,53 & 9,39 & 0,49 & 0,921 \\
\hline & Bimaleolar $(\mathrm{cm})$ & 7,60 & 0,23 & 7,41 & 0,40 & 0,265 \\
\hline
\end{tabular}

$\overline{\mathrm{X}}=$ Promedio, SD: Desviación estándar,

* = Diferencia estadísticamente significativa

Fuente: autores, 2013 
Tabla 3. Comparación de los resultados del somatotipo con los publicados en otros estudios

\begin{tabular}{lcccc}
\cline { 2 - 5 } & ruta & SDD & pista & SDD \\
\hline Resultados del estudio & $2,0-4,1-2,8$ & NA & $2,7-5,3-1,9$ & NA \\
White et al., 1982 & $2,0-4,3-2,7$ & 0,22 & $2,4-5,1-2,4$ & 0,62 \\
Foley et al., 1989 & $2,1-4,8-3,5$ & 0,99 & $2,2-6,9-1,4$ & 1,75 \\
McLean y Parker, 1989 & $2,1-4,7-2,9$ & 0,62 & $2,3-5,3-2,3$ & 0,57 \\
Martínez et al., 1993 & $2,4-4,8-2,3$ & 0,95 & & \\
Padilla et al., 2004 & $3,2-3,7-2,5$ & 1,30 & & \\
\hline
\end{tabular}

SDD: Distancia de dispersión somatotípica, que representa la distancia entre dos somatotipos; es significativa cuando su valor es superior a 2,0. NA: No aplica.

Fuente: autores, 2013

Figura 1. Diagrama de cajas y bigotes con los resultados comparativos de pliegues en los deportistas evaluados

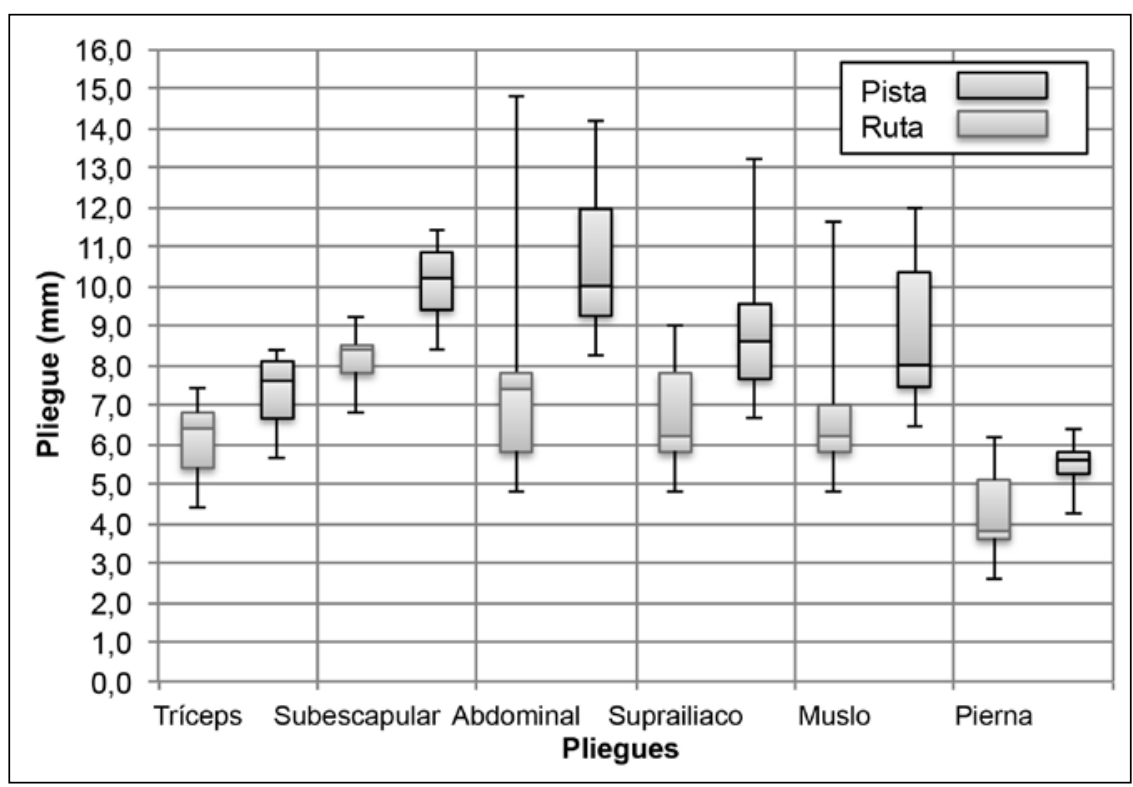

Fuente: autores, 2013 
Figura 2. Diagrama de cajas y bigotes con los resultados comparativos de los perímetros de los deportistas evaluados

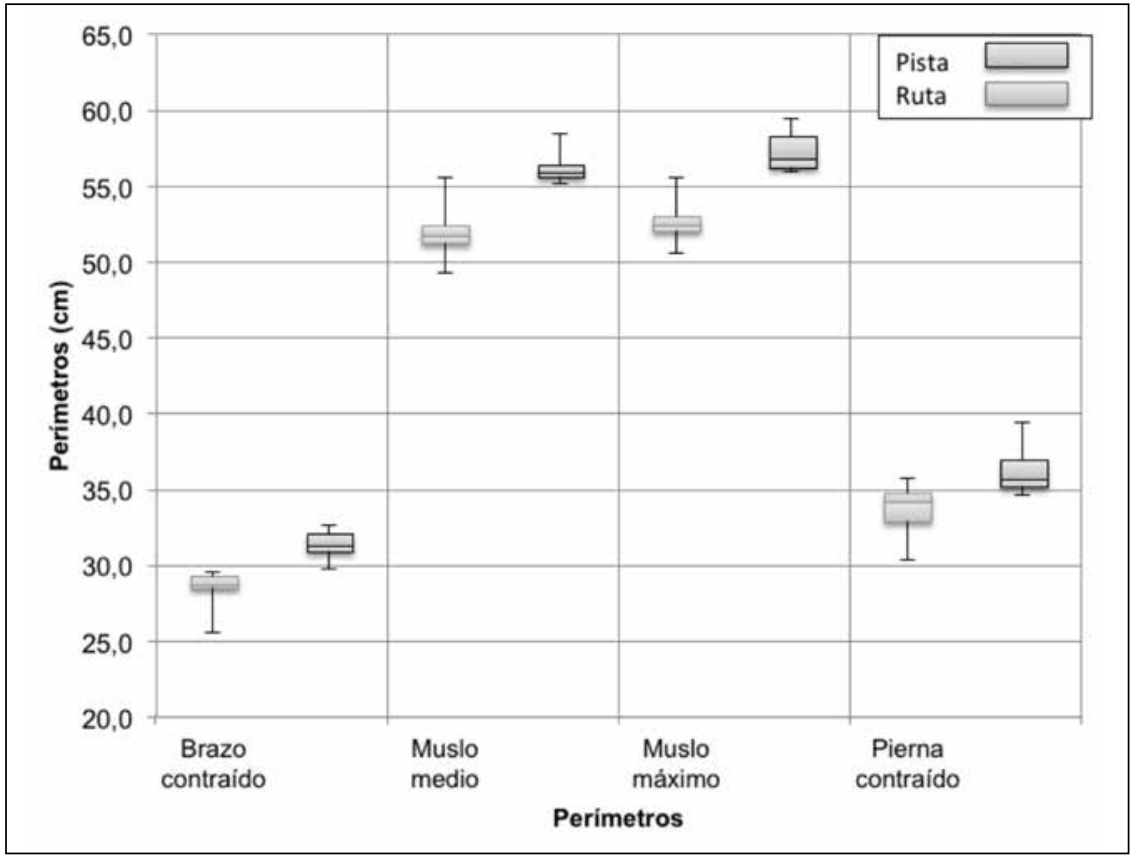

Fuente: autores, 2013

Figura 3. Representación gráfica de la diferencia en composición de los ciclistas de pista y ruta, ${ }^{*}=\mathrm{p}<0,05$

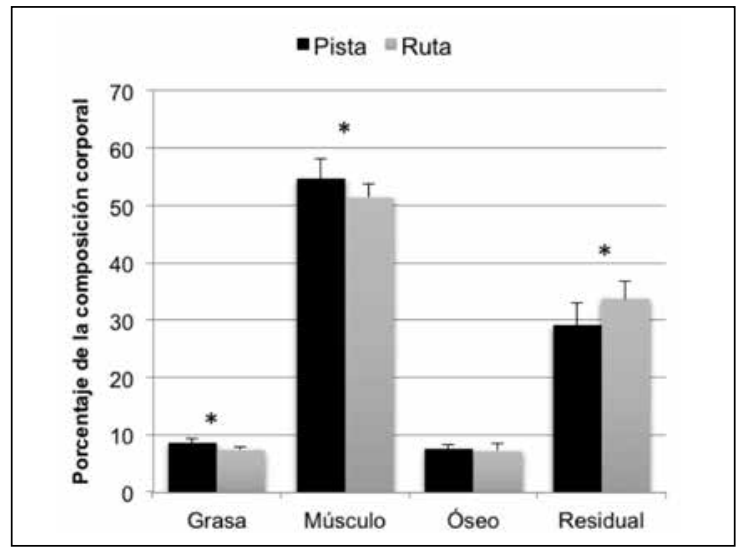

Fuente: autores, 2013 
Figura 4. Carta somatoscópica con la representación gráfica de los ciclistas de pista y ruta evaluados

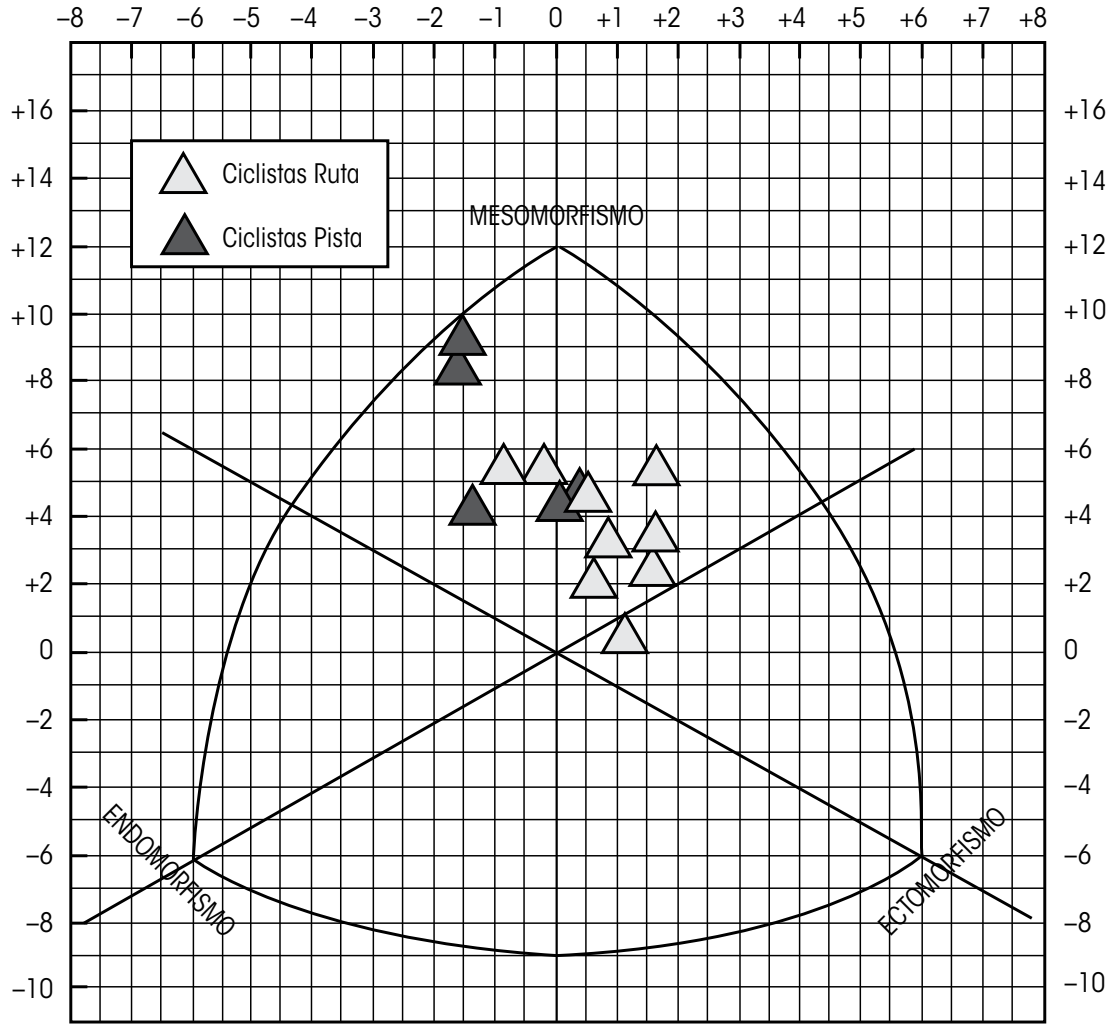

Fuente: autores, 2013 
Figura 5. Carta somatoscópica en la que se encuentran graficadas la dispersión del somatotipo (SDI) para cada modalidad deportiva y la distancia de dispersión entre los dos grupos (SDD)

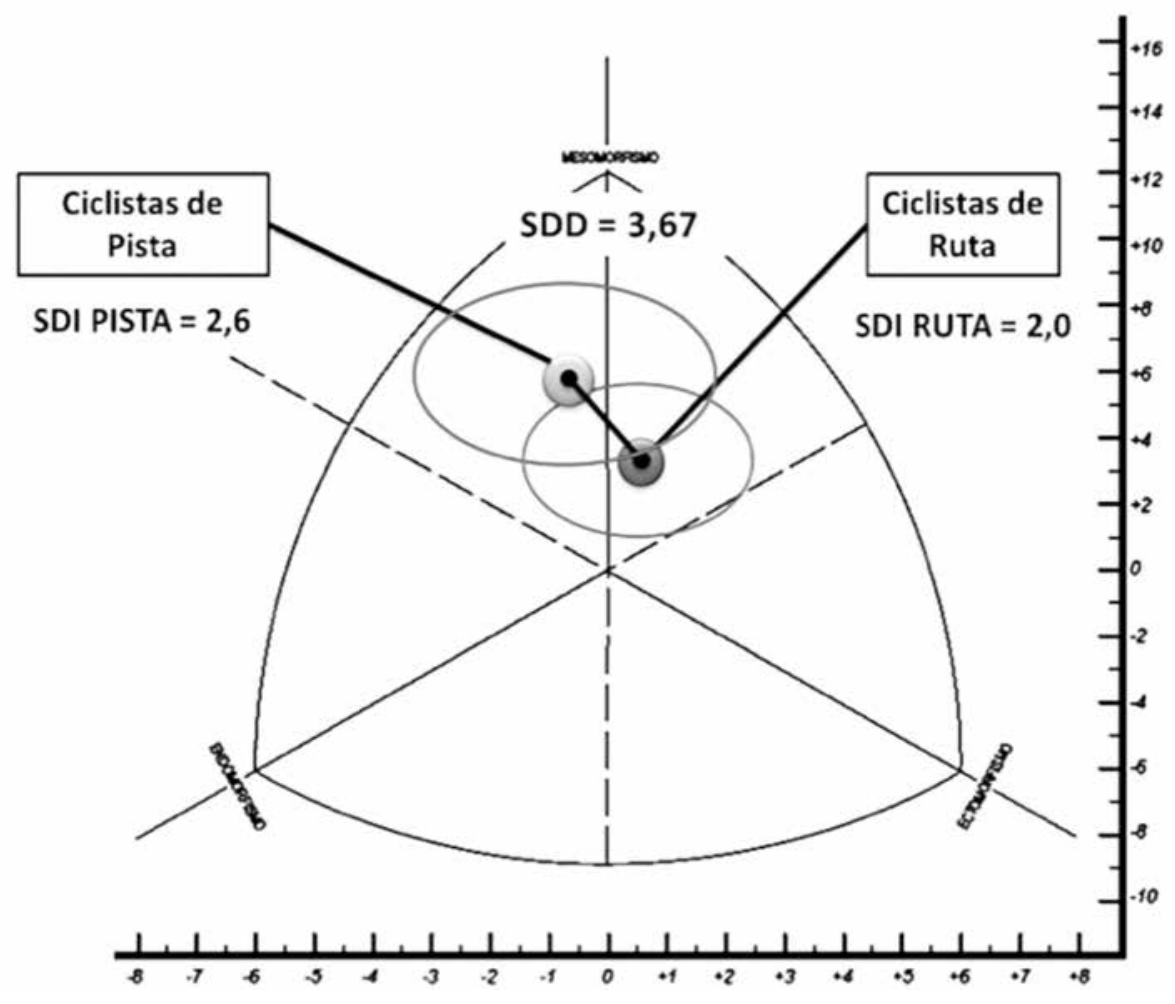

Fuente: autores, 2013 